\title{
On boundary behavior of mappings with finite distortion in the plane
}

\author{
Denis Kovtonyuk, Igor Petkov and Vladimir Ryazanov
}

July 9, 2018

\begin{abstract}
In the present paper, it was studied the boundary behavior of the so-called lower $Q$-homeomorphisms in the plane that are a natural generalization of the quasiconformal mappings. In particular, it was found a series of effective conditions on the function $Q(z)$ for a homeomorphic extension of the given mappings to the boundary by prime ends. The developed theory is applied to mappings with finite distortion by Iwaniec, also to solutions of the Beltrami equations, as well as to finitely bi-Lipschitz mappings that a far-reaching extension of the known classes of isometric and quasiisometric mappings.
\end{abstract}

\section{Mathematics Subject Classification: Primary 30C62, 30C65, 30D40, 37E30. Secondary 35A16, 35A23, 35J67, 35J70, 35J75.}

Key words: Boundary behavior, prime ends, mappings of finite distortion, lower $Q$-homeomorphisms, finitely bi-Lipschitz mappings, isometric and quasiisometric mappings, Beltrami equations.

\section{Introduction}

The problem of the boundary behavior is one of the central topics of the theory of quasiconformal mappings and their generalizations. During the last years, it is intensively studied various classes of mappings with finite distortion in a natural way generalizing conformal, quasiconformal and quasiregular mappings, see e.g. many references in the monographs [16], [18, [20] and [48]. In this case, as it was earlier, the main geometric approach in the modern mapping theory is the method of moduli, see, e.g., the monographs [16], [48], [55], [64], [87], [88] and 90 . 
One of the main foundations in the present paper is the theory of the boundary behavior by prime ends for the so-called lower $Q$-homeomorphisms developed here in the first part. The second one is Theorem 3.1 on solutions of the Beltrami equations in [24] that can be reformulated in the following way.

Theorem 1.1. Let $f$ be a homeomorphism with finite distortion in a domain $D \subseteq \mathbb{C}$. Then $f$ is a lower Q-homeomorphism at each point $z_{0} \in \bar{D}$ with $Q=K_{f}$.

As usual, $K_{f}(z)$ denotes the dilatation of the mapping $f$ at $z$, namely,

$$
K_{f}(z)=\frac{\left|f_{z}\right|+\left|f_{\bar{z}}\right|}{\left|f_{z}\right|-\left|f_{\bar{z}}\right|}
$$

if $f_{z} \neq 0,1$ if $f_{z}=0$ and $\infty$ otherwise, where $f_{\bar{z}}=\left(f_{x}+i f_{y}\right) / 2, f_{z}=$ $\left(f_{x}-i f_{y}\right) / 2, z=x+i y$, and $f_{x}$ and $f_{y}$ are partial derivatives of $f$ in $x$ and $y$, correspondingly.

Here we follow Caratheodory [5] in the definition of the prime ends for bounded finitely connected domains in $\mathbb{C}$ and refer readers to Chapter 9 in [7], see also [1] and [54] for the history of the question.

Reducing this case to Theorem 9.3 in [7] for simple connected domains, we obtain the following basic fact for the theory of lower $Q$-homeomorphisms.

Lemma 1.1. Each prime end $P$ of a bounded finitely connected domain $D$ in $\mathbb{C}$ contains a chain of cross-cuts $\sigma_{m}$ lying on circles $S\left(z_{0}, r_{m}\right)=\{z \in \mathbb{C}$ : $\left.\left|z-z_{0}\right|=r_{m}\right\}$ with $z_{0} \in \partial D$ and $r_{m} \rightarrow 0$ as $m \rightarrow \infty$.

Remark 1.1. As known, every bounded finitely connected domain $D$ in $\mathbb{C}$ can be mapped by a conformal mapping $g_{0}$ onto the so-called circular domain $D_{0}$ whose boundary consists of a finite collection of mutually disjoint circles and isolated points, see, e.g., Theorem V.6.2 in [15]. Moreover, isolated singular points of bounded conformal mappings are removable by Theorem 1.2 in [7] due to Weierstrass. Hence isolated points of $\partial D$ correspond to isolated points of $\partial D_{0}$ and inversely.

Reducing this case to the Caratheodory theorem, see, e.g., Theorem 9.4 in [7] for simple connected domains, we have a natural one-to-one correspondence between points of $\partial D_{0}$ and prime ends of the domain $D$. Denote by 
$\bar{D}_{P}$ the completion of $D$ with its prime ends and determine in $\bar{D}_{P}$ the metric $\rho_{0}\left(p_{1}, p_{2}\right)=\left|\widetilde{g_{0}}\left(p_{1}\right)-\widetilde{g_{0}}\left(p_{2}\right)\right|$ where $\widetilde{g_{0}}$ is the extension of $g_{0}$ to $\bar{D}_{P}$ mentioned above.

If $g_{*}$ is another conformal mapping of the domain $D$ on a circular domain $D_{*}$, then the corresponding metric $\rho_{*}\left(p_{1}, p_{2}\right)=\left|\widetilde{g_{*}}\left(p_{1}\right)-\widetilde{g_{*}}\left(p_{2}\right)\right|$ generates the same convergence in $\bar{D}_{P}$ as the metric $\rho_{0}$ because $g_{0} \circ g_{*}^{-1}$ is a conformal mapping between the domains $D_{*}$ and $D_{0}$ that is extended to a homeomorphism between $\overline{D_{*}}$ and $\overline{D_{0}}$. It is easy to see the latter by applying Theorems 1.2 and 3.2 in [7], see also Lemma 5.3 and Corollary 5.2 in [19], correspondingly, Lemma 6.5 and Corollary 6.12 in [48]. Consequently, the given metrics induce the same topology in the space $\bar{D}_{P}$ that we will call the topology of prime ends.

This topology can be also described in inner terms of the domain $D$, see, e.g., Section 9.5 in [7], however, we prefer the definition through the metrics because it is more clear, more convenient and it is important for us just metrizability of $\bar{D}_{P}$. Note also that the space $\bar{D}_{P}$ for every bounded finitely connected domain $D$ in $\mathbb{C}$ with the given topology is compact because the closure of the circular domain $D_{0}$ is a compact space and by the construction $\widetilde{g}_{0}: \bar{D}_{P} \rightarrow \overline{D_{0}}$ is a homeomorphism.

Later on, we mean the continuity of mappings $f: \bar{D}_{P} \rightarrow{\overline{D^{\prime}}}_{P}$ just with respect to this topology.

\section{On lower $Q$-homeomorphisms}

A continuous mapping $\gamma$ of an open subset $\Delta$ of the real axis $\mathbb{R}$ or a circle into $D$ is called a dashed line, see, e.g., Section 6.3 in [48]. Recall that every open set $\Delta$ in $\mathbb{R}$ consists of a countable collection of mutually disjoint intervals. This is the motivation for the term.

Given a family $\Gamma$ of dashed lines $\gamma$ in complex plane $\mathbb{C}$, a Borel function $\varrho: \mathbb{C} \rightarrow[0, \infty]$ is called admissible for $\Gamma$, write $\varrho \in \operatorname{adm} \Gamma$, if

$$
\int_{\gamma} \varrho d s \geqslant 1
$$


for every $\gamma \in \Gamma$. The (conformal) modulus of $\Gamma$ is the quantity

$$
M(\Gamma)=\inf _{\varrho \in \operatorname{adm} \Gamma} \int_{\mathbb{C}} \varrho^{2}(z) d m(z)
$$

where $d m(z)$ corresponds to the Lebesgue measure in $\mathbb{C}$. We say that a property $P$ holds for a.e. (almost every) $\gamma \in \Gamma$ if a subfamily of all lines in $\Gamma$ for which $P$ fails has the modulus zero. Later on, we also say that a Lebesgue measurable function $\varrho: \mathbb{C} \rightarrow[0, \infty]$ is extensively admissible for $\Gamma$, write $\varrho \in \operatorname{ext} \operatorname{adm} \Gamma$, if (2.1) holds for a.e. $\gamma \in \Gamma$, see, e.g., Section 9.2 in [48].

The following concept was motivated by Gehring's ring definition of quasiconformality in [9] and first introduced in the paper [26]. Given bounded domains $D$ and $D^{\prime}$ in $\mathbb{C}, z_{0} \in \bar{D}$, and a measurable function $Q: \mathbb{C} \rightarrow(0, \infty)$, we say that a homeomorphism $f: D \rightarrow D^{\prime}$ is a lower $Q$-homeomorphism at the point $z_{0}$ if

$$
M\left(f \Sigma_{\varepsilon}\right) \geqslant \inf _{\varrho \in \operatorname{extadm} \Sigma_{\varepsilon}} \int_{D \cap R_{\varepsilon}} \frac{\varrho^{2}(z)}{Q(z)} d m(z)
$$

for every $\operatorname{ring} R_{\varepsilon}=\left\{z \in \overline{\mathbb{C}}: \varepsilon<\left|z-z_{0}\right|<\varepsilon_{0}\right\}, \quad \varepsilon \in\left(0, \varepsilon_{0}\right), \varepsilon_{0} \in\left(0, d_{0}\right)$, where $d_{0}=\sup \left|z-z_{0}\right|$, and $\Sigma_{\varepsilon}$ denotes the family of all intersections of the circles $S\left(z_{0}, r \in D=\left\{z \in \mathbb{C}:\left|z-z_{0}\right|=r\right\}, \quad r \in\left(\varepsilon, \varepsilon_{0}\right)\right.$, with the domain $D$. We also say that a homeomorphism $f: D \rightarrow D^{\prime}$ is a lower $Q$-homeomorphism if $f$ is a lower $Q$-homeomorphism at every point $x_{0} \in \bar{D}$.

Recall the criterion for homeomorphisms in $\mathbb{C}$ to be lower $Q$-homeomorphisms, see Theorem 2.1 in [27], correspondingly, Theorem 9.2 in [48].

Proposition 2.1. Let $D$ and $D^{\prime}$ be bounded domains in $\mathbb{C}, z_{0} \in \bar{D}$, and $Q: \mathbb{C} \rightarrow(0, \infty)$ be a measurable function. A homeomorphism $f: D \rightarrow D^{\prime}$ is a lower $Q$-homeomorphism at $z_{0}$ if and only if

$$
M\left(f \Sigma_{\varepsilon}\right) \geqslant \int_{\varepsilon}^{\varepsilon_{0}} \frac{d r}{\|Q\|\left(z_{0}, r\right)} \quad \forall \varepsilon \in\left(0, \varepsilon_{0}\right), \varepsilon_{0} \in\left(0, d_{0}\right),
$$

where $d_{0}=\sup _{z \in D}\left|z-z_{0}\right|$ and $\|Q\|\left(z_{0}, r\right)$ is the $L_{1}$-norm of $Q$ over $D \cap S\left(z_{0}, r\right)$. 


\section{On regular domains}

Recall first of all the following topological notion. A domain $D \subset \mathbb{C}$ is said to be locally connected at a point $z_{0} \in \partial D$ if, for every neighborhood $U$ of the point $z_{0}$, there is a neighborhood $V \subseteq U$ of $z_{0}$ such that $V \cap D$ is connected. Note that every Jordan domain $D$ in $\mathbb{C}$ is locally connected at each point of $\partial D$, see e.g. [92], p. 66.

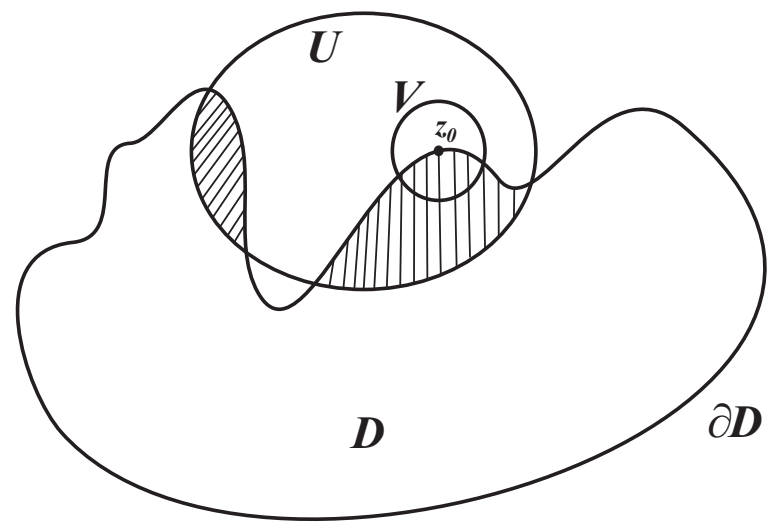

We say that $\partial D$ is weakly flat at a point $z_{0} \in \partial D$ if, for every neighborhood $U$ of the point $z_{0}$ and every number $P>0$, there is a neighborhood $V \subset U$ of $z_{0}$ such that

$$
M(\Delta(E, F ; D)) \geqslant P
$$

for all continua $E$ and $F$ in $D$ intersecting $\partial U$ and $\partial V$. Here and later on, $\Delta(E, F ; D)$ denotes the family of all paths $\gamma:[a, b] \rightarrow \overline{\mathbb{C}}$ connecting $E$ and $F$ in $D$, i.e. $\gamma(a) \in E, \gamma(b) \in F$ and $\gamma(t) \in D$ for all $t \in(a, b)$. We say that the boundary $\partial D$ is weakly flat if it is weakly flat at every point in $\partial D$.

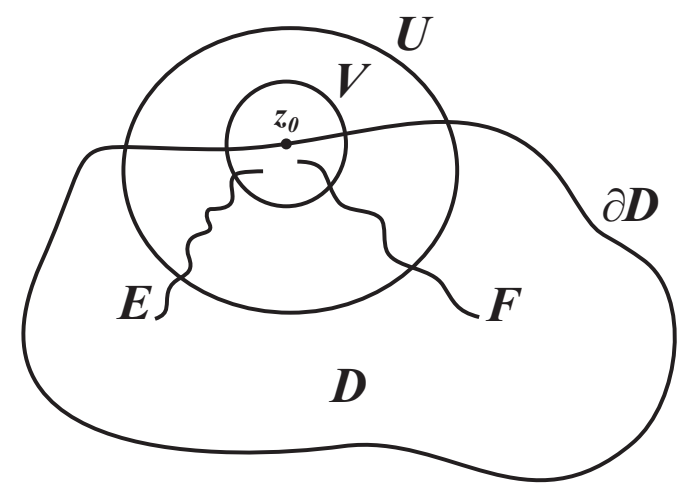

We also say that a point $z_{0} \in \partial D$ is strongly accessible if, for every neighborhood $U$ of the point $z_{0}$, there exist a compactum $E$ in $D$, a neighborhood 
$V \subset U$ of $z_{0}$ and a number $\delta>0$ such that

$$
M(\Delta(E, F ; D)) \geqslant \delta
$$

for all continua $F$ in $D$ intersecting $\partial U$ and $\partial V$. We say that the boundary $\partial D$ is strongly accessible if every point $z_{0} \in \partial D$ is strongly accessible.

Here, in the definitions of strongly accessible and weakly flat boundaries, one can take as neighborhoods $U$ and $V$ of a point $z_{0}$ only balls (closed or open) centered at $z_{0}$ or only neighborhoods of $z_{0}$ in another fundamental system of neighborhoods of $z_{0}$.

It is easy to see that if a domain $D$ in $\mathbb{C}$ is weakly flat at a point $z_{0} \in \partial D$, then the point $z_{0}$ is strongly accessible from $D$. Moreover, it was proved by us that if a domain $D$ in $\mathbb{C}$ is weakly flat at a point $z_{0} \in \partial D$, then $D$ is locally connected at $z_{0}$, see, e.g., Lemma 5.1 in [27] or Lemma 3.15 in [48].

The notions of strong accessibility and weak flatness at boundary points of a domain in $\mathbb{C}$ defined in [26] are localizations and generalizations of the corresponding notions introduced in [46]-[47], cf. with the properties $P_{1}$ and $P_{2}$ by Väisälä in [88] and also with the quasiconformal accessibility and the quasiconformal flatness by Näkki in [53]. Many theorems on a homeomorphic extension to the boundary of quasiconformal mappings and their generalizations are valid under the condition of weak flatness of boundaries. The condition of strong accessibility plays a similar role for a continuous extension of the mappings to the boundary. In particular, recently we have proved the following significant statements, see either Theorem 10.1 (Lemma 6.1) in [27] or Theorem 9.8 (Lemma 9.4) in [48].

Proposition 3.1. Let $D$ and $D^{\prime}$ be bounded domains in $\mathbb{C}, Q: D \rightarrow(0, \infty)$ a measurable function and $f: D \rightarrow D^{\prime}$ a lower $Q$-homeomorphism in $\partial D$. Suppose that the domain $D$ is locally connected on $\partial D$ and that the domain $D^{\prime}$ has a (strongly accessible) weakly flat boundary. If

$$
\int_{0}^{\delta\left(z_{0}\right)} \frac{d r}{\|Q\|\left(z_{0}, r\right)}=\infty \quad \forall z_{0} \in \partial D
$$


for some $\delta\left(z_{0}\right) \in\left(0, d\left(z_{0}\right)\right)$ where $d\left(z_{0}\right)=\sup _{z \in D}\left|z-z_{0}\right|$ and

$$
\|Q\|\left(z_{0}, r\right)=\int_{S\left(z_{0}, r\right)} Q(z) d s
$$

then $f$ has a (continuous) homeomorphic extension $\bar{f}: \bar{D} \rightarrow \overline{D^{\prime}}$.

A domain $D \subset \mathbb{C}$ is called a quasiextremal distance domain, abbr. QED-domain, see [10], if

$$
M(\Delta(E, F ; \overline{\mathbb{C}}) \leqslant K \cdot M(\Delta(E, F ; D))
$$

for some $K \geqslant 1$ and all pairs of nonintersecting continua $E$ and $F$ in $D$.

It is well known, see, e.g., Theorem 10.12 in [88], that

$$
M(\Delta(E, F ; \mathbb{C})) \geqslant \frac{2}{\pi} \log \frac{R}{r}
$$

for any sets $E$ and $F$ in $\mathbb{C}$ intersecting all the circles $S\left(z_{0}, \rho\right), \rho \in(r, R)$. Hence a QED-domain has a weakly flat boundary. One example in [48], Section 3.8, shows that the inverse conclusion is not true even among simply connected plane domains.

A domain $D \subset \mathbb{C}$ is called a uniform domain if each pair of points $z_{1}$ and $z_{2} \in D$ can be joined with a rectifiable curve $\gamma$ in $D$ such that

$$
s(\gamma) \leqslant a \cdot\left|z_{1}-z_{2}\right|
$$

and

$$
\min _{i=1,2} s\left(\gamma\left(z_{i}, z\right)\right) \leqslant b \cdot d(z, \partial D)
$$

for all $z \in \gamma$ where $\gamma\left(z_{i}, z\right)$ is the portion of $\gamma$ bounded by $z_{i}$ and $z$, see [51]. It is known that every uniform domain is a QED-domain but there exist QEDdomains that are not uniform, see [10]. Bounded convex domains and bounded domains with smooth boundaries are simple examples of uniform domains and, consequently, QED-domains as well as domains with weakly flat boundaries.

In the mapping theory and in the theory of differential equations, it is often applied the so-called Lipschitz domains whose boundaries are weakly flat. 
Recall first that a map $\varphi: X \rightarrow Y$ between metric spaces $X$ and $Y$ is said to be Lipschitz provided $\operatorname{dist}\left(\varphi\left(x_{1}\right), \varphi\left(x_{2}\right)\right) \leqslant M \cdot \operatorname{dist}\left(x_{1}, x_{2}\right)$ for some $M<\infty$ and for all $x_{1}$ and $x_{2} \in X$. The map $\varphi$ is called bi-Lipschitz if, in addition, $M^{*} \operatorname{dist}\left(x_{1}, x_{2}\right) \leqslant \operatorname{dist}\left(\varphi\left(x_{1}\right), \varphi\left(x_{2}\right)\right)$ for some $M^{*}>0$ and for all $x_{1}$ and $x_{2} \in X$. Later on, $X$ and $Y$ are subsets of $\mathbb{C}$ with the Euclidean distance.

It is said that a domain $D$ in $\mathbb{C}$ is Lipschitz if every point $z_{0} \in \partial D$ has a neighborhood $U$ that can be mapped by a bi-Lipschitz homeomorphism $\varphi$ onto the unit disk $\mathbb{D}$ in $\mathbb{C}$ in such a way that $\varphi(\partial D \cap U)$ is the intersection of $\mathbb{D}$ with a coordinate axes and $f\left(z_{0}\right)=0$, see, e.g., [55]. Note that bi-Lipschitz homeomorphisms are quasiconformal and hence the Lipschitz domains have weakly flat boundaries.

\section{On continuous extension of lower $Q$-homeomorphisms}

Lemma 4.1. Let $D$ and $D^{\prime}$ be bounded finitely connected domains in $\mathbb{C}$ and let $f: D \rightarrow D^{\prime}$ be a lower $Q$-homeomorphis. If

$$
\int_{0}^{\varepsilon_{0}} \frac{d r}{\|Q\|\left(z_{0}, r\right)}=\infty \quad \forall z_{0} \in \partial D
$$

where $0<\varepsilon_{0}<d_{0}=\sup _{z \in D}\left|z-z_{0}\right|$ and

$$
\|Q\|\left(z_{0}, r\right)=\int_{S\left(z_{0}, r\right)} Q d s
$$

then $f$ can be extended to a continuous mapping of $\bar{D}_{P}$ onto ${\overline{D^{\prime}}}_{P}$.

Proof. With no loss of generality we may assume that $D^{\prime}$ is a circular domain and, thus, $\overline{D^{\prime}} P=\overline{D^{\prime}}$. By metrizability and compactness of $\overline{D^{\prime}}$, it suffices to prove that, for each prime end $P$ of the domain $D$, the cluster set

$L=C(P, f):=\left\{\zeta \in \mathbb{C}: \zeta=\lim _{n \rightarrow \infty} f\left(z_{n}\right), z_{n} \rightarrow P, z_{n} \in D, n=1,2, \ldots\right\}$ consists of a single point $\zeta_{0} \in \partial D^{\prime}$.

Note that $L \neq \varnothing$ by compactness of the set $\overline{D^{\prime}}$, and $L$ is a subset of $\partial D^{\prime}$, see, e.g., Proposition 2.5 in [68] or Proposition 13.5 in [48]. Let us assume that there 
is at least two points $\zeta_{0}$ and $\zeta_{*} \in L$. Set $U=B\left(\zeta_{0}, r_{0}\right)=\left\{\zeta \in \mathbb{C}:\left|\zeta-\zeta_{0}\right|<r_{0}\right\}$ where $0<r_{0}<\left|\zeta_{*}-\zeta_{0}\right|$.

Let $\sigma_{k}, k=1,2, \ldots$, be a chain of cross-cuts of $D$ in the prime end $P$ lying on circles $S_{k}=S\left(z_{0}, r_{k}\right)$ from Lemma 1.1 where $z_{0} \in \partial D$. Let $D_{k}, k=1,2, \ldots$ be the domains associated with $\sigma_{k}$. Then there exist points $\zeta_{k}$ and $\zeta_{k}^{*}$ in the domains $D_{k}^{\prime}=f\left(D_{k}\right)$ such that $\left|\zeta_{0}-\zeta_{k}\right|<r_{0}$ and $\left|\zeta_{0}-\zeta_{k}^{*}\right|>r_{0}$ and, moreover, $\zeta_{k} \rightarrow \zeta_{0}$ and $\zeta_{k}^{*} \rightarrow \zeta_{*}$ as $k \rightarrow \infty$. Let $C_{k}$ be continuous curves joining $\zeta_{k}$ and $\zeta_{k}^{*}$ in $D_{k}^{\prime}$. Note that by the construction $\partial U \cap C_{k} \neq \varnothing$.

By the condition of strong accessibility of the point $\zeta_{0}$, there is a continuum $E \subset D^{\prime}$ and a number $\delta>0$ such that

$$
M\left(\Delta\left(E, C_{k} ; D^{\prime}\right)\right) \geqslant \delta
$$

for all large enough $k$.

Without loss of generality, we may assume that the latter condition holds for all $k=1,2, \ldots$ Note that $C=f^{-1}(E)$ is a compact subset of $D$ and hence $\varepsilon_{0}=\operatorname{dist}\left(z_{0}, C\right)>0$. Again, with no loss of generality, we may assume that $r_{k}<\varepsilon_{0}$ for all $k=1,2, \ldots$.

Let $\Gamma_{m}$ be a family of all continuous curves in $D \backslash D_{m}$ joining the circle $S_{0}=$ $S\left(z_{0}, \varepsilon_{0}\right)$ and $\overline{\sigma_{m}}, m=1,2, \ldots$. Note that by the construction $C_{k} \subset D_{k}^{\prime} \subset D_{m}^{\prime}$ for all $m \leqslant k$ and, thus, by the principle of minorization $M\left(f\left(\Gamma_{m}\right)\right) \geqslant \delta$ for all $m=1,2, \ldots$

On the other hand, the quantity $M\left(f\left(\Gamma_{m}\right)\right)$ is equal to the capacity of the condenser in $D^{\prime}$ with facings $\overline{D_{m}^{\prime}}$ and $\overline{f\left(D \backslash B_{0}\right)}$ where $B_{0}=B\left(z_{0}, \varepsilon_{0}\right)$, see, e.g., [79]. Thus, by the principle of minorization and Theorem 3.13 in [93]

$$
M\left(f\left(\Gamma_{m}\right)\right) \leqslant \frac{1}{\left(f\left(\Sigma_{m}\right)\right)}
$$

where $\Sigma_{m}$ is the collection of all intersections of the domain $D$ and the spheres $S\left(z_{0}, \rho\right), \rho \in\left(r_{m}, \varepsilon_{0}\right)$, because $f\left(\Sigma_{m}\right) \subset \Sigma\left(f\left(S_{m}\right), f\left(S_{0}\right)\right)$ where $\Sigma\left(f\left(S_{m}\right), f\left(S_{0}\right)\right)$ consists of all closed subsets of $D^{\prime}$ separating $f\left(S_{m}\right)$ and $f\left(S_{0}\right)$. Finally, by the condition (4.1) we obtain that $M\left(f\left(\Gamma_{m}\right)\right) \rightarrow 0$ as $m \rightarrow \infty$.

The obtained contradiction disproves the assumption that the cluster set $C(P, f)$ consists of more than one point. 


\section{Extension of inverse maps of lower $Q$-homeomorphisms}

The base for the proof on extending the inverse mappings of lower $Q$-homeomorphism by prime ends in the plane is the following fact on the cluster sets.

Lemma 5.1. Let $D$ and $D^{\prime}$ be bounded finitely connected domains in $\mathbb{C}$, and let $f: D \rightarrow D^{\prime}$ be a lower $Q$-homeomorphism. Denote by $P_{1}$ and $P_{2}$ different prime ends of the domain $D$ and by $\sigma_{m}, m=1,2, \ldots$, a chain of cross-cuts in the prime end $P_{1}$ from Lemma 1.1, lying on circles $S\left(z_{1}, r_{m}\right), z_{1} \in \partial D$, with associated domains $d_{m}$. Suppose that $Q$ is integrable over $D \cap S\left(z_{1}, r\right)$ for a set $E$ of numbers $r \in(0, \delta)$ of a positive linear measure where $\delta=r_{m_{0}}$ and $m_{0}$ is a minimal number such that the domain $d_{m_{0}}$ does not contain sequences of points converging to $P_{2}$. If $\partial D^{\prime}$ is weakly flat, then

$$
C\left(P_{1}, f\right) \cap C\left(P_{2}, f\right)=\varnothing .
$$

Note that in view of metrizability of the completion $\bar{D}_{P}$ of the domain $D$ with prime ends, see Remark 1.1, the number $m_{0}$ in Lemma 5.1 always exists.

Proof. Let us choose $\varepsilon \in(0, \delta)$ such that $E_{0}:=\{r \in E: r \in(\varepsilon, \delta)\}$ has a positive linear measure. Such a choice is possible in view of subadditivity of the linear measure and the exhaustion $E=\cup E_{m}$ where $E_{m}=\{r \in E: r \in$ $(1 / m, \delta)\}, m=1,2, \ldots$. Note that by Proposition 2.1

$$
M\left(f\left(\Sigma_{\varepsilon}\right)\right)>0
$$

where $\Sigma_{\varepsilon}$ is the family of all surfaces $D(r)=D \cap S\left(z_{1}, r\right), r \in(\varepsilon, \delta)$.

Let us assume that $C_{1} \cap C_{2} \neq \varnothing$ where $C_{i}=C\left(P_{i}, f\right), i=1,2$. By the construction there is $m_{1}>m_{0}$ such that $\sigma_{m_{1}}$ lies on the circle $S\left(z_{1}, r_{m_{1}}\right)$ with $r_{m_{1}}<\varepsilon$. Let $d_{0}=d_{m_{1}}$ and $d_{*} \subseteq D \backslash d_{m_{0}}$ be a domain associated with a chain of cross-cuts in the prime end $P_{2}$. Let $\zeta_{0} \in C_{1} \cap C_{2}$. Choose $r_{0}>0$ such that $S\left(\zeta_{0}, r_{0}\right) \cap f\left(d_{0}\right) \neq \varnothing$ and $S\left(\zeta_{0}, r_{0}\right) \cap f\left(d_{*}\right) \neq \varnothing$.

Set $\Gamma=\Delta\left(\overline{d_{0}}, \overline{d_{*}} ; D\right)$. Correspondingly (5.2), by the principle of minorization and Theorem 3.13 in [93],

$$
M(f(\Gamma)) \leqslant \frac{1}{M\left(f\left(\Sigma_{\varepsilon}\right)\right)}<\infty .
$$


Let $M_{0}>M(f(\Gamma))$ be a finite number. By the condition of the lemma, $\partial D^{\prime}$ is weakly flat and hence there is $r_{*} \in\left(0, r_{0}\right)$ such that

$$
M\left(\Delta\left(E, F ; D^{\prime}\right)\right) \geqslant M_{0}
$$

for all continua $E$ and $F$ in $D^{\prime}$ intersecting the circles $S\left(\zeta_{0}, r_{0}\right)$ and $S\left(\zeta_{0}, r_{*}\right)$. However, these circles can be joined by continuous curves $c_{1}$ and $c_{2}$ in the domains $f\left(d_{0}\right)$ and $f\left(d_{*}\right)$, correspondingly, and, in particular, for these curves

$$
M_{0} \leqslant M\left(\Delta\left(c_{1}, c_{2} ; D^{\prime}\right)\right) \leqslant M(f(\Gamma)) .
$$

The obtained contradiction disproves the assumption that $C_{1} \cap C_{2} \neq \varnothing$.

Theorem 5.1. Let $D$ and $D^{\prime}$ be bounded finitely connected domains in $\mathbb{C}$ and $f: D \rightarrow D^{\prime}$ be a lower $Q$-homeomorphism with $Q \in L^{1}(D)$. Then $f^{-1}$ can be extended to a continuous mapping of ${\overline{D^{\prime}}}_{P}$ onto $\bar{D}_{P}$.

Proof. By Remark 1.1, we may assume with no loss of generality that $D^{\prime}$ is a circular domain, $\overline{D^{\prime}} P=\overline{D^{\prime}} ; C\left(\zeta_{0}, f^{-1}\right) \neq \varnothing$ for every $\zeta_{0} \in \partial D^{\prime}$ because $\bar{D}_{P}$ is metrizable and compact. Moreover, $C\left(\zeta_{0}, f^{-1}\right) \cap D=\varnothing$, see, e.g., Proposition 2.5 in [68] or Proposition 13.5 in [48].

Let us assume that there is at least two different prime ends $P_{1}$ and $P_{2}$ in $C\left(\zeta_{0}, f^{-1}\right)$. Then $\zeta_{0} \in C\left(P_{1}, f\right) \cap C\left(P_{2}, f\right)$ and, thus, (5.1) does not hold. Let $z_{1} \in \partial D$ be a point corresponding to $P_{1}$ from Lemma 1.1. Note that

$$
E=\left\{r \in(0, \delta):\left.Q\right|_{D \cap S\left(z_{1}, r\right)} \in L^{1}\left(D \cap S\left(z_{1}, r\right)\right)\right\}
$$

has a positive linear measure for every $\delta>0$ by the Fubini theorem, see, e.g., [77], because $Q \in L^{1}(D)$. The obtained contradiction with Lemma 5.1] shows that $C\left(\zeta_{0}, f^{-1}\right)$ contains only one prime end of $D$.

Thus, we have the extension $g$ of $f^{-1}$ to $\overline{D^{\prime}}$ such that $C\left(\partial D^{\prime}, f^{-1}\right) \subseteq \bar{D}_{P} \backslash D$. Really $C\left(\partial D^{\prime}, f^{-1}\right)=\bar{D}_{P} \backslash D$. Indeed, if $P_{0}$ is a prime end of $D$, then there is a sequence $z_{n}$ in $D$ being convergent to $P_{0}$. We may assume without loss of generality that $z_{n} \rightarrow z_{0} \in \partial D$ and $f\left(z_{n}\right) \rightarrow \zeta_{0} \in \partial D^{\prime}$ because $\bar{D}$ and $\overline{D^{\prime}}$ are compact. Hence $P_{0} \in C\left(\zeta_{0}, f^{-1}\right)$.

Finally, let us show that the extended mapping $g: \bar{D}^{\prime} \rightarrow \bar{D}_{P}$ is continuous. Indeed, let $\zeta_{n} \rightarrow \zeta_{0}$ in $\overline{D^{\prime}}$. If $\zeta_{0} \in D^{\prime}$, then the statement is obvious. If $\zeta_{0} \in \partial D^{\prime}$, 
then take $\zeta_{n}^{*} \in D^{\prime}$ such that $\left|\zeta_{n}-\zeta_{n}^{*}\right|<1 / n$ and $\rho\left(g\left(\zeta_{n}\right), g\left(\zeta_{n}^{*}\right)\right)<1 / n$ where $\rho$ is one of the metrics in Remark 1.1. Note that by the construction $g\left(\zeta_{n}^{*}\right) \rightarrow g\left(\zeta_{0}\right)$ because $\zeta_{n}^{*} \rightarrow \zeta_{0}$. Consequently, $g\left(\zeta_{n}\right) \rightarrow g\left(\zeta_{0}\right)$, too.

Theorem 5.2. Let $D$ and $D^{\prime}$ be bounded finitely connected domains in $\mathbb{C}$. If $f: D \rightarrow D^{\prime}$ is a lower Q-homeomorphism with condition (4.1), then $f^{-1}$ can be extended to a continuous mapping of ${\overline{D^{\prime}}}_{P}$ onto $\bar{D}_{P}$.

Proof. Indeed, by Lemma 9.2 in [27] or Lemma 9.6 in [48], condition (4.1) implies that

$$
\int_{0}^{\delta} \frac{d r}{\|Q\|\left(z_{0}, r\right)}=\infty \quad \forall z_{0} \in \partial D \quad \forall \delta \in\left(0, \varepsilon_{0}\right)
$$

and, thus, the set

$$
E=\left\{r \in(0, \delta):\left.Q\right|_{D \cap S\left(z_{0}, r\right)} \in L^{1}\left(D \cap S\left(z_{0}, r\right)\right)\right\}
$$

has a positive linear measure for all $z_{0} \in \partial D$ and all $\delta \in\left(0, \varepsilon_{0}\right)$. The rest of arguments is perfectly similar to one in the proof of Theorem 5.1 .

\section{On functions of finite mean oscillation}

Recall that a real-valued function $u$ in a domain $D$ in $\mathbb{C}$ is said to be of bounded mean oscillation in $D$, abbr. $u \in \operatorname{BMO}(D)$, or simply $u \in \mathrm{BMO}$ if $u \in$ $L_{\text {loc }}^{1}(D)$ and

$$
\|u\|_{*}:=\sup _{B} \frac{1}{|B|} \int_{B}\left|u(z)-u_{B}\right| d m(z)<\infty,
$$

where the supremum is taken over all discs $B$ in $D, d m(z)$ corresponds to the Lebesgue measure in $\mathbb{C}$ and $u_{B}$ is the average of $u$ over $B$. We write $u \in \mathrm{BMO}_{\text {loc }}(D)$ if $u \in \mathrm{BMO}(U)$ for every relatively compact subdomain $U$ of $D$ (we also write $\mathrm{BMO}$ or $\mathrm{BMO}_{\text {loc }}$ if it is clear from the context what $D$ is).

The class BMO was introduced by John and Nirenberg in the paper [23] and soon became an important concept in harmonic analysis, partial differential equations and related areas, see, e.g., [17] and [60]. 
A function $\varphi$ in BMO is said to have vanishing mean oscillation (abbreviated as $\varphi \in \mathbf{V M O}$ ), if the supremum in (6.1) taken over all disks $B$ in $D$ with $|B|<\varepsilon$ converges to 0 as $\varepsilon \rightarrow 0$. VMO was introduced by Sarason in [78]. Note that a large number of papers are devoted to the existence, uniqueness and properties of solutions for various kinds of differential equations and, in particular, of elliptic type with coefficients of the class VMO, see, e.g., [6, 21, 49, 56, 61].

Following the paper [19], see also the monographs [16] and [48], we say that a function $\varphi: D \rightarrow \mathbb{R}$ has finite mean oscillation at a point $z_{0} \in D$ if

$$
\varlimsup_{\varepsilon \rightarrow 0} f_{B\left(z_{0}, \varepsilon\right)}\left|\varphi(z)-\widetilde{\varphi}_{\varepsilon}\left(z_{0}\right)\right| d m(z)<\infty,
$$

where

$$
\widetilde{\varphi}_{\varepsilon}\left(z_{0}\right)=f_{B\left(z_{0}, \varepsilon\right)} \varphi(z) d m(z)
$$

is the mean value of the function $\varphi(z)$ over the disk $B\left(z_{0}, \varepsilon\right)$. Note that the condition (6.2) includes the assumption that $\varphi$ is integrable in some neighborhood of the point $z_{0}$. We say also that a function $\varphi: D \rightarrow \mathbb{R}$ is of finite mean oscillation in $D$, abbr. $\varphi \in \operatorname{FMO}(D)$ or simply $\varphi \in \operatorname{FMO}$, if $\varphi \in \operatorname{FMO}\left(z_{0}\right)$ for all points $z_{0} \in D$. We write $\varphi \in \operatorname{FMO}(\bar{D})$ if $\varphi$ is given in a domain $G$ in $\mathbb{C}$ such that $\bar{D} \subset G$ and $\varphi \in \operatorname{FMO}\left(z_{0}\right)$ for all $z_{0} \in \bar{D}$.

The following statement is obvious by the triangle inequality.

Proposition 6.1. If, for a collection of numbers $\varphi_{\varepsilon} \in \mathbb{R}, \varepsilon \in\left(0, \varepsilon_{0}\right]$,

$$
\varlimsup_{\varepsilon \rightarrow 0} f_{B\left(z_{0}, \varepsilon\right)}\left|\varphi(z)-\varphi_{\varepsilon}\right| d m(z)<\infty,
$$

then $\varphi$ is of finite mean oscillation at $z_{0}$.

In particular choosing in Proposition [6.1, $\varphi_{\varepsilon} \equiv 0, \varepsilon \in\left(0, \varepsilon_{0}\right]$, we obtain the following.

Corollary 6.1. If, for a point $z_{0} \in D$,

$$
\varlimsup_{\varepsilon \rightarrow 0} f_{B\left(z_{0}, \varepsilon\right)}|\varphi(z)| d m(z)<\infty,
$$

then $\varphi$ has finite mean oscillation at $z_{0}$. 
Recall that a point $z_{0} \in D$ is called a Lebesgue point of a function $\varphi$ : $D \rightarrow \mathbb{R}$ if $\varphi$ is integrable in a neighborhood of $z_{0}$ and

$$
\lim _{\varepsilon \rightarrow 0} f_{B\left(z_{0}, \varepsilon\right)}\left|\varphi(z)-\varphi\left(z_{0}\right)\right| d m(z)=0 .
$$

It is known that, almost every point in $D$ is a Lebesgue point for every function $\varphi \in L^{1}(D)$. Thus, we have by Proposition 6.1 the following corollary showing that the FMO condition is natural.

Corollary 6.2. Every locally integrable function $\varphi: D \rightarrow \mathbb{R}$ has a finite mean oscillation at almost every point in $D$.

Remark 6.1. Note that the function $\varphi(z)=\log (1 /|z|)$ belongs to BMO in the unit disk $\mathbb{D}$ in $\mathbb{C}$, see, e.g., [60], p. 5, and hence also to FMO. However, $\widetilde{\varphi}_{\varepsilon}(0) \rightarrow \infty$ as $\varepsilon \rightarrow 0$, showing that condition (6.5) is only sufficient but not necessary for a function $\varphi$ to be of finite mean oscillation at $z_{0}$. Clearly, $\mathrm{BMO}(D) \subset \mathrm{BMO}_{\text {loc }}(D) \subset \mathrm{FMO}(D)$ and as well-known $\mathrm{BMO}_{\text {loc }} \subset L_{\text {loc }}^{p}$ for all $p \in[1, \infty)$, see, e.g., [23]. However, FMO is not a subclass of $L_{\mathrm{loc}}^{p}$ for any $p>1$ but only $L_{\text {loc }}^{1}$, see examples in [48], p. 211. Thus, the class FMO is essentially wider than $\mathrm{BMO}_{\text {loc }}$.

Versions of the next lemma has been first proved for the class BMO in the planar case in [72] and then in the space case in [47]. For the FMO case, see the papers [19] and [70] and also the monographs [16] and [48].

Lemma 6.1. Let $D$ be a domain in $\mathbb{C}$ and let $\varphi: D \rightarrow \mathbb{R}$ be a non-negative function of the class $\operatorname{FMO}\left(z_{0}\right)$ for some $z_{0} \in D$. Then

$$
\int_{\varepsilon<\left|z-z_{0}\right|<\varepsilon_{0}} \frac{\varphi(z) d m(z)}{\left(\left|z-z_{0}\right| \log \frac{1}{\left|z-z_{0}\right|}\right)^{2}}=O\left(\log \log \frac{1}{\varepsilon}\right) \quad \text { as } \quad \varepsilon \rightarrow 0
$$

for some $\varepsilon_{0} \in\left(0, \delta_{0}\right)$ where $\delta_{0}=\min \left(e^{-e}, d_{0}\right), d_{0}=\sup _{z \in D}\left|z-z_{0}\right|$.

\section{Homeomorphic extension of lower $Q$-homeomorphisms}

Combining Lemma 4.1 and Theorem 5.2, we obtain the next conclusion. 
Theorem 7.1. Let $D$ and $D^{\prime}$ be bounded finitely connected domains in $\mathbb{C}$ and let $f: D \rightarrow D^{\prime}$ be a lower $Q$-homeomorphism with

$$
\int_{0}^{\varepsilon_{0}} \frac{d r}{\|Q\|\left(z_{0}, r\right)}=\infty \quad \forall z_{0} \in \partial D
$$

where $0<\varepsilon_{0}<d_{0}=\sup _{z \in D}\left|z-z_{0}\right|$ and

$$
\|Q\|\left(z_{0}, r\right)=\int_{S\left(z_{0}, r\right)} Q d s
$$

Then $f$ can be extended to a homeomorphism of $\bar{D}_{P}$ onto ${\overline{D^{\prime}}}_{P}$.

Corollary 7.1. In particular, the conclusion of Theorem 7.1 holds if

$$
q_{z_{0}}(r)=O\left(\log \frac{1}{r}\right) \quad \forall z_{0} \in \partial D
$$

as $r \rightarrow 0$ where $q_{z_{0}}(r)$ is the average of $Q$ over the circle $\left|z-z_{0}\right|=r$.

Using Lemma 2.2 in [70], see also Lemma 7.4 in [48], by Theorem 7.1 we obtain the following general lemma that, in turn, makes possible to obtain new criteria in a great number.

Lemma 7.1. Let $D$ and $D^{\prime}$ be bounded finitely connected domains in $\mathbb{C}$ and let $f: D \rightarrow D^{\prime}$ be a lower $Q$-homeomorphism. Suppose that

$$
\int_{D\left(z_{0}, \varepsilon\right)} Q(z) \cdot \psi_{z_{0}, \varepsilon}^{2}\left(\left|z-z_{0}\right|\right) d m(z)=o\left(I_{x_{0}}^{2}(\varepsilon)\right) \quad \forall z_{0} \in \partial D
$$

as $\varepsilon \rightarrow 0$ where $D\left(z_{0}, \varepsilon\right)=\left\{z \in D: \varepsilon<\left|z-z_{0}\right|<\varepsilon_{0}\right\}$ for $0<\varepsilon_{0}<$ $d\left(z_{0}\right)=\sup _{z \in D}\left|z-z_{0}\right|$ and where $\psi_{z_{0}, \varepsilon}(t):(0, \infty) \rightarrow[0, \infty], \varepsilon \in\left(0, \varepsilon_{0}\right)$, is a two-parameter family of measurable functions such that

$$
0<I_{z_{0}}(\varepsilon):=\int_{\varepsilon}^{\varepsilon_{0}} \psi_{z_{0}, \varepsilon}(t) d t<\infty \quad \forall \varepsilon \in\left(0, \varepsilon_{0}\right) .
$$

Then $f$ can be extended to a homeomorphism of $\bar{D}_{P}$ onto ${\overline{D^{\prime}}}_{P}$.

Remark 7.1. Note that (7.3) holds, in particular, if

$$
\int_{B\left(z_{0}, \varepsilon_{0}\right)} Q(z) \cdot \psi^{2}\left(\left|z-z_{0}\right|\right) d m(z)<\infty \quad \forall z_{0} \in \partial D
$$


where $B\left(z_{0}, \varepsilon_{0}\right)=\left\{z \in D:\left|z-z_{0}\right|<\varepsilon_{0}\right\}$ and where $\psi(t):(0, \infty) \rightarrow[0, \infty]$ is a measurable function such that $I_{z_{0}}(\varepsilon) \rightarrow \infty$ as $\varepsilon \rightarrow 0$. In other words, for the extendability of $f$ to a homeomorphism of $\bar{D}_{P}$ onto ${\overline{D^{\prime}}}_{P}$, it suffices the integrals in (7.4) to be convergent for some nonnegative function $\psi(t)$ that is locally integrable on $(0, \infty)$ but it has a non-integrable singularity at zero.

Note also that it is not only Lemma 7.1 follows from Theorem 7.1 but, inversely, Theorem 7.1 follows from Lemma 7.1, too. Indeed, for the function

$$
\psi_{z_{0}}(t)=\left\{\begin{array}{lr}
1 /\|Q\|\left(z_{0}, t\right), & t \in\left(0, \varepsilon_{0}\right), \\
0, & t \in\left[\varepsilon_{0}, \infty\right),
\end{array}\right.
$$

we have by the Fubini theorem that

$$
\int_{S\left(z_{0}, \varepsilon\right)} Q(z) \cdot \psi_{z_{0}}^{2}\left(\left|z-z_{0}\right|\right) d m(z)=\int_{\varepsilon}^{\varepsilon_{0}} \frac{d r}{\|Q\|\left(z_{0}, r\right)} .
$$

Thus, Theorem 7.1 is equivalent to Lemma 7.1 but each of them is sometimes more convenient for applications than another one.

Choosing in Lemma $7.1 \psi(t):=\frac{1}{t \log 1 / t}$ and applying Lemma 6.1, we obtain the next result.

Theorem 7.2. Let $D$ and $D^{\prime}$ be bounded finitely connected domains in $\mathbb{C}$ and let $f: D \rightarrow D^{\prime}$ be a lower $Q$-homeomorphism. If $Q(z)$ has finite mean oscillation at every point $z_{0} \in \partial D$, then $f$ can be extended to a homeomorphism of $\bar{D}_{P}$ onto ${\overline{D^{\prime}}}_{P}$.

Corollary 7.2. In particular, the conslusion of Theorem 7.2 holds if

$$
\varlimsup_{\varepsilon \rightarrow 0} f_{B\left(z_{0}, \varepsilon\right)} Q(z) d m(z)<\infty \quad \forall z_{0} \in \partial D
$$

Corollary 7.3. The conslusion of Theorem 7.2 holds if every point $z_{0} \in \partial D$ is a Lebesgue point of the function $Q: \mathbb{C} \rightarrow(0, \infty)$.

The next statement also follows from Lemma 7.1 under the choice $\psi(t)=1 / t$.

Theorem 7.3. Let $D$ and $D^{\prime}$ be bounded finitely connected domains in $\mathbb{C}$ 
and $f: D \rightarrow D^{\prime}$ be a lower $Q$-homeomorphism. If, for some $\varepsilon_{0}=\varepsilon\left(z_{0}\right)>0$,

$$
\int_{\varepsilon<\left|z-z_{0}\right|<\varepsilon_{0}} Q(z) \frac{d m(z)}{\left|z-z_{0}\right|^{2}}=o\left(\left[\log \frac{1}{\varepsilon}\right]^{2}\right) \quad \forall z_{0} \in \partial D
$$

as $\varepsilon \rightarrow 0$, then $f$ can be extended to a homeomorphism of $\bar{D}_{P}$ onto ${\overline{D^{\prime}}}_{P}$.

Remark 7.2. Choosing in Lemma 7.1 the function $\psi(t)=1 /(t \log 1 / t)$ instead of $\psi(t)=1 / t$, (7.8) can be replaced by the more weak condition

$$
\int_{\varepsilon<\left|z-z_{0}\right|<\varepsilon_{0}} \frac{Q(z) d m(z)}{\left|z-z_{0}\right| \log \frac{1}{\left|z-z_{0}\right|}}=o\left(\left[\log \log \frac{1}{\varepsilon}\right]^{2}\right)
$$

and (7.2) by the condition

$$
q_{z_{0}}(r)=o\left(\log \frac{1}{r} \log \log \frac{1}{r}\right) .
$$

Of course, we could to give here the whole scale of the corresponding condition of the logarithmic type using suitable functions $\psi(t)$.

Theorem 7.1 has a magnitude of other fine consequences, for instance:

Theorem 7.4. Let $D$ and $D^{\prime}$ be bounded finitely connected domains in $\mathbb{C}$ and let $f: D \rightarrow D^{\prime}$ be a lower $Q$-homeomorphism with

$$
\int_{D} \Phi(Q(z)) d m(z)<\infty
$$

for a nondecreasing convex function $\Phi:[0, \infty) \rightarrow[0, \infty)$ such that

$$
\int_{\delta_{*}}^{\infty} \frac{d \tau}{\tau \Phi^{-1}(\tau)}=\infty
$$

for $\delta_{*}>\Phi(0)$. Then $f$ is extended to a homeomorphism of $\bar{D}_{P}$ onto ${\overline{D^{\prime}}}_{P}$.

Indeed, by Theorem 3.1 and Corollary 3.2 in [76], (7.11) and (77.12) imply (7.1) and, thus, Theorem 7.4 is a direct consequence of Theorem 7.1 .

Corollary 7.4. In particular, the conclusion of Theorem 7.2 holds if

$$
\int_{D} e^{\alpha Q(z)} d m(z)<\infty
$$


for some $\alpha>0$.

Remark 7.3. By Theorem 2.1 in [76], see also Proposition 2.3 in [71], (7.12) is equivalent to every of the conditions from the following series:

$$
\begin{gathered}
\int_{\delta}^{\infty} H^{\prime}(t) \frac{d t}{t}=\infty, \quad \delta>0, \\
\int_{\delta}^{\infty} \frac{d H(t)}{t}=\infty, \quad \delta>0, \\
\int_{\delta}^{\infty} H(t) \frac{d t}{t^{2}}=\infty, \quad \delta>0, \\
\int_{0}^{\Delta} H\left(\frac{1}{t}\right) d t=\infty, \quad \Delta>0, \\
\int_{\delta_{0}}^{\infty} \frac{d \eta}{H^{-1}(\eta)}=\infty, \quad \delta_{0}>H(0),
\end{gathered}
$$

where

$$
H(t)=\log \Phi(t)
$$

Here the integral in (7.15) is understood as the Lebesgue-Stieltjes integral and the integrals in (7.14) and (7.16) $-($ (7.18) as the ordinary Lebesgue integrals.

It is necessary to give one more explanation. From the right hand sides in the conditions (7.14) $-(7.18)$ we have in mind $+\infty$. If $\Phi(t)=0$ for $t \in\left[0, t_{*}\right]$, then $H(t)=-\infty$ for $t \in\left[0, t_{*}\right]$ and we complete the definition $H^{\prime}(t)=0$ for $t \in\left[0, t_{*}\right]$. Note, the conditions (17.15) and (7.16) exclude that $t_{*}$ belongs to the interval of integrability because in the contrary case the left hand sides in (7.15) and (7.16) are either equal to $-\infty$ or indeterminate. Hence we may assume in (7.14)-(7.17) that $\delta>t_{0}$, correspondingly, $\Delta<1 / t_{0}$ where $t_{0}:=\sup _{\Phi(t)=0} t$, set $t_{0}=0$ if $\Phi(0)>0$. 
The most interesting of the above conditions is (7.16) that can be rewritten in the form:

$$
\int_{\delta}^{\infty} \log \Phi(t) \frac{d t}{t^{2}}=\infty .
$$

Finally, note that if a domain $D$ in $\mathbb{C}$ is locally connected on its boundary, then there is a natural one-to-one correspondence between prime ends of $D$ and boundary points of $D$. Thus, if $D$ and $D^{\prime}$ are in addition locally connected on their boundaries in theorems of Sections 4 and 5 , then $f$ is extended to a homeomorphism of $\bar{D}$ onto $\overline{D^{\prime}}$. We obtained before it similar results when $\partial D^{\prime}$ was weakly flat which is a more strong condition than local connectivity of $D^{\prime}$ on its boundary, see, e.g., [24] and [25].

As known, every Jordan domain $D$ in $\mathbb{C}$ is locally connected on its boundary, see, e.g., [92], p. 66. It is easy to see, the latter implies that every bounded finitely connected domain $D$ in $\mathbb{C}$ whose boundary consists of mutually disjoint Jordan curves and isolated points is also locally connected on its boundary.

Inversely, every bounded finitely connected domain $D$ in $\mathbb{C}$ which is locally connected on its boundary has a boundary consisting of mutually disjoint Jordan curves and isolated points. Indeed, every such a domain $D$ can be mapped by a conformal mapping $f$ onto the so-called circular domain $D_{*}$ bounded by a finite collection of mutually disjoint circles and isolated points, see, e.g., Theorem V.6.2 in [15], that is extended to a homeomorphism of $\bar{D}$ onto $\overline{D_{*}}$.

Note also that, under every homeomorphism $f$ between domains $D$ and $D^{\prime}$ in $\overline{\mathbb{C}}$, there is a natural one-to-one correspondence between components of their boundaries $\partial D$ and $\partial D^{\prime}$, see, e.g., Lemma 5.3 in [19] or Lemma 6.5 in [48]. Thus, if a bounded domain $D$ in $\mathbb{C}$ is finitely connected and $D^{\prime}$ is bounded, then $D^{\prime}$ is finitely connected, too.

\section{Boundary behavior of mappings with finite distortion}

Recall that a homeomorphism $f$ between domains $D$ and $D^{\prime}$ in $\mathbb{R}^{n}, n \geqslant 2$, is called of finite distortion if $f \in W_{\mathrm{loc}}^{1,1}$ and

$$
\left\|f^{\prime}(x)\right\|^{n} \leqslant K(x) \cdot J_{f}(x)
$$


with some a.e. finite function $K$ where $f^{\prime}(x)$ denotes the Jacobian matrix of $f$ at $x \in D$ if it exists, $J_{f}(x)=\operatorname{det} f^{\prime}(x)$ is the Jacobian of $f$ at $x$, and $\left\|f^{\prime}(x)\right\|$ is the operator norm of $f^{\prime}(x)$, i.e.,

$$
\left\|f^{\prime}(x)\right\|=\max \left\{\left|f^{\prime}(x) h\right|: h \in \mathbb{R}^{n},|h|=1\right\} .
$$

In the complex plane $\left\|f^{\prime}\right\|=\left|f_{z}\right|+\left|f_{\bar{z}}\right|$ and $J_{f}=\left|f_{z}\right|^{2}-\left|f_{\bar{z}}\right|^{2}$, i.e., (8.1) is equivalent to the condition that $K_{f}(z)<\infty$ a.e., see (1.1).

First this notion was introduced on the plane for $f \in W_{\text {loc }}^{1,2}$ in the work [22]. Later on, this condition was replaced by $f \in W_{\text {loc }}^{1,1}$ but with the additional condition $J_{f} \in L_{\text {loc }}^{1}$ in the monograph [20]. The theory of the mappings with finite distortion had many successors, see many relevant references in the monographs [16], [18], [20] and [48]. They had as predecessors of the mappings with bounded distortion, see [63], and also [89], in other words, the quasiregular mappings, see, e.g., [17], [49], [64] and [90]. They are also closely connected to the earlier mappings with the bounded Dirichlet integral, see, e.g., the monographs [44], [83] and [84], and the mappings quasiconformal in the mean which had a rich history, see, e.g., [2]-[4], [11]-[14], [30]-[43], [57]-[59], [65]-[67], [81]-[82], [85]-[86], [94] and [95].

Note that the above additional condition $J_{f} \in L_{\text {loc }}^{1}$ in the definition of the mappings with finite distortion can be omitted for homeomorphisms. Indeed, for each homeomorphism $f$ between domains $D$ and $D^{\prime}$ in $\mathbb{R}^{n}$ with the first partial derivatives a.e. in $D$, there is a set $E$ of the Lebesgue measure zero such that $f$ satisfies $(N)$-property by Lusin on $D \backslash E$ and

$$
\int_{A} J_{f}(x) d m(x)=|f(A)|
$$

for every Borel set $A \subset D \backslash E$, see, e.g., 3.1.4, 3.1.8 and 3.2.5 in [8].

On the basis of Theorem 1.1 and the corresponding results on lower $Q$-homeomorphisms in Sections 5 and 6, we obtain the following conclusions on the boundary behavior of mappings with finite distortion.

Theorem 8.1. Let $D$ and $D^{\prime}$ be bounded finitely connected domains in $\mathbb{C}$ 
and $f: D \rightarrow D^{\prime}$ be a homeomorphism of finite distortion with $K_{f} \in L^{1}(D)$. Then $f^{-1}$ can be extended to a continuous mapping of ${\overline{D^{\prime}}}_{P}$ onto $\bar{D}_{P}$.

It is sufficient to assume in Theorem 8.1 that $K_{f}$ is integrable only in a neighborhood of $\partial D$ and even more weak conditions on $K_{f}$ due to Lemma 5.1 .

However, any degree of integrability of $K_{f}$ cannot guarantee a continuous extension of the direct mappings $f$ to the boundary, see an example in the proof of Proposition 6.3 in [48]. Conditions for it have a perfectly different nature. The principal related result is the following.

Theorem 8.2. Let $D$ and $D^{\prime}$ be bounded finitely connected domains in $\mathbb{C}$, $f: D \rightarrow D^{\prime}$ be a homeomorphism of finite distortion with condition

$$
\int_{0}^{\varepsilon_{0}} \frac{d r}{\left\|K_{f}\right\|\left(z_{0}, r\right)}=\infty \quad \forall z_{0} \in \partial D
$$

where $0<\varepsilon_{0}<d_{0}=\sup _{z \in D}\left|z-z_{0}\right|$ and

$$
\left\|K_{f}\right\|\left(z_{0}, r\right)=\int_{S\left(z_{0}, r\right)} K_{f} d s
$$

Then $f$ can be extended to a homeomorphism of $\bar{D}_{P}$ onto ${\overline{D^{\prime}}}_{P}$.

Here we assume that $K_{f}$ is extended by zero outside of the domain $D$.

Corollary 8.1. In particular, the conclusion of Theorem 8.2 holds if

$$
k_{z_{0}}(r)=O\left(\log \frac{1}{r}\right) \quad \forall z_{0} \in \partial D
$$

as $r \rightarrow 0$ where $k_{z_{0}}(r)$ is the average of $K_{f}$ over the circle $\left|z-z_{0}\right|=r$.

Lemma 8.1. Let $D$ and $D^{\prime}$ be bounded finitely connected domains in $\mathbb{C}$ and let $f: D \rightarrow D^{\prime}$ be a homeomorphism with finite distortion. Suppose that

$$
\int_{\varepsilon<\left|z-z_{0}\right|<\varepsilon_{0}} K_{f}(z) \cdot \psi_{z_{0}, \varepsilon}^{2}\left(\left|z-z_{0}\right|\right) d m(z)=o\left(I_{z_{0}}^{2}(\varepsilon)\right) \quad \forall z_{0} \in \partial D
$$


as $\varepsilon \rightarrow 0$ where $0<\varepsilon_{0}<\sup _{z \in D}\left|z-z_{0}\right|$ and where $\psi_{z_{0}, \varepsilon}(t):(0, \infty) \rightarrow[0, \infty]$, $\varepsilon \in\left(0, \varepsilon_{0}\right)$, is a two-parameter family of measurable functions such that

$$
0<I_{z_{0}}(\varepsilon):=\int_{\varepsilon}^{\varepsilon_{0}} \psi_{z_{0}, \varepsilon}(t) d t<\infty \quad \forall \varepsilon \in\left(0, \varepsilon_{0}\right) .
$$

Then $f$ can be extended to a homeomorphism of $\bar{D}_{P}$ onto ${\overline{D^{\prime}}}_{P}$.

Theorem 8.3. Let $D$ and $D^{\prime}$ be bounded finitely connected domains in $\mathbb{C}$ and let $f: D \rightarrow D^{\prime}$ be a homeomorphism with finite distortion. If $K_{f}(z)$ has finite mean oscillation at every point $z_{0} \in \partial D$, then $f$ can be extended to $a$ homeomorphism of $\bar{D}_{P}$ onto ${\overline{D^{\prime}}}_{P}$.

In fact, here it is sufficient $K_{f}(z)$ to have a dominant of finite mean oscillation in a neighborhood of every point $z_{0} \in \partial D$.

Corollary 8.2. In particular, the conclusion of Theorem 8.3 holds if

$$
\varlimsup_{\varepsilon \rightarrow 0} f_{B\left(z_{0}, \varepsilon\right)} K_{f}(z) d m(z)<\infty \quad \forall z_{0} \in \partial D
$$

Theorem 8.4. Let $D$ and $D^{\prime}$ be bounded finitely connected domains in $\mathbb{C}$ and let $f: D \rightarrow D^{\prime}$ be a homeomorphism with finite distortion such that

$$
\int_{\varepsilon<\left|z-z_{0}\right|<\varepsilon_{0}} K_{f}(z) \frac{d m(z)}{\left|z-z_{0}\right|^{2}}=o\left(\left[\log \frac{1}{\varepsilon}\right]^{2}\right) \quad \forall z_{0} \in \partial D
$$

Then $f$ can be extended to a homeomorphism of $\bar{D}_{P}$ onto ${\overline{D^{\prime}}}_{P}$.

Remark 8.1. Choosing in Lemma 7.1 the function $\psi(t)=1 /(t \log 1 / t)$ instead of $\psi(t)=1 / t$, (8.9) can be replaced by the more weak condition

$$
\int_{\varepsilon<\left|z-z_{0}\right|<\varepsilon_{0}} \frac{K_{f}(z) d m(z)}{\left(\left|z-z_{0}\right| \log \frac{1}{\left|z-z_{0}\right|}\right)^{2}}=o\left(\left[\log \log \frac{1}{\varepsilon}\right]^{2}\right) .
$$

Of course, we could give here the whole scale of the corresponding conditions of the logarithmic type using suitable functions $\psi(t)$. In particular, condition (8.6) can be weakened with the help of Theorem 8.2 by condition

$$
k_{z_{0}}(r)=O\left(\log \frac{1}{r} \log \log \frac{1}{r}\right)
$$


Theorem 8.2 has a magnitude of other fine consequences, for instance:

Theorem 8.5. Let $D$ and $D^{\prime}$ be bounded finitely connected domains in $\mathbb{C}$ and let $f: D \rightarrow D^{\prime}$ be a homeomorphism with finite distortion such that

$$
\int_{D} \Phi\left(K_{f}(z)\right) d m(z)<\infty
$$

for a nondecreasing convex function $\Phi:[0, \infty) \rightarrow[0, \infty)$ such that

$$
\int_{\delta_{*}}^{\infty} \frac{d \tau}{\tau \Phi^{-1}(\tau)}=\infty
$$

for $\delta_{*}>\Phi(0)$. Then $f$ is extended to a homeomorphism of $\bar{D}_{P}$ onto ${\overline{D^{\prime}}}_{P}$.

Corollary 8.3. In particular, the conclusion of Theorem 7.4 holds if

$$
\int_{D} e^{\alpha K_{f}(z)} d m(z)<\infty
$$

for some $\alpha>0$.

Remark 8.2. Note that the condition (8.13) is not only sufficient but also necessary for a cotinuous extension to the boundary of the mappings $f$ with integral restrictions of the form (8.12), see, e.g., Theorem 5.1 and Remark 5.1 in [29].

\section{Boundary behavior of finitely bi-Lipschitz mappings}

Given a domain $D \subseteq \mathbb{C}$, following Section 5 in [28], see also Section 10.6 in [48], we say that a mapping $f: D \rightarrow \mathbb{C}$ is finitely bi-Lipschitz if

$$
0<l(z, f) \leqslant L(z, f)<\infty \quad \forall z \in D
$$

where

$$
L(z, f)=\limsup _{\zeta \rightarrow z} \frac{|f(\zeta)-f(z)|}{|\zeta-z|}
$$

and

$$
l(z, f)=\liminf _{\zeta \rightarrow z} \frac{|f(\zeta)-f(z)|}{|\zeta-z|} .
$$


The class of finitely bi-Lipschitz homeomorphisms is a natural generalization of the well-known classes of isometries and quasi-isometries. However, they are generally speaking are not of finite distortion by Iwaniec.

By the classic Stepanov theorem, see [80], see also [45], we obtain from the right hand inequality in (9.1) that finitely bi-Lipschitz mappings are differentiable a.e. and from the left hand inequality in (9.1) that $J_{f}(x) \neq 0$ a.e. Moreover, such mappings have $(N)$-property with respect to each Hausdorff measure, see, e.g., either Lemma 5.3 in [28] or Lemma 10.6 [48]. In particular, they are ACL but generally speaking are not in the class $W_{\text {loc }}^{1,1}$.

However, by Corollary 5.15 in [28] and Corollary 10.10 in [48]:

Lemma 9.1. Every finitely bi-Lipschitz homeomorphism $f: \Omega \rightarrow \mathbb{C}$ is a lower $Q$-homeomorphism with $Q=K_{f}$.

Corollary 9.1. All results on homeomorphisms with finite distortion in Section 8 are valid for finitely bi-Lipschitz homeomorphisms.

All these results for finitely bi-Lipschitz homeomorphisms are perfectly similar to the corresponding results for homeomorphisms with finite distortion in Section 8. Hence we will not formulate them in the explicit form here.

The results of this paper can be applied to the theory of boundary value problems for the Beltrami equations including equations of the second kind that take an important part in many problems of mathematical physics.

\section{References}

[1] Adamowicza T., Buörna A., Buörna J., Shanmugalingamb N. Prime ends for domains in metric spaces // Advances in Mathematics. - 2013. - 238. - P. 459 - 505.

[2] Ahlfors L. On quasiconformal mappings // J. Anal. Math. - 1953/1954. - 3. - P. 1-58.

[3] Belinski P.P. General properties of quasiconformal mappings. - Novosibirsk: Izdat. "Nauka" Sibirsk. Otdel., 1974 [in Russian].

[4] Biluta P.A. Extremal problems for mappings quasiconformal in the mean // Sib. Mat. Zh. - 1965. - 6. - P. 717-726 [in Russian].

[5] Caratheodory C. Über die Begrenzung der einfachzusammenhängender Gebiete // Math. Ann. - 1913. - 73. - P. 323 - 370. 
[6] Chiarenza F., Frasca M., Longo P. $W^{2, p}$-solvability of the Dirichlet problem for nondivergence elliptic equations with VMO coefficients // Trans. Amer. Math. Soc. - 1993. 336, no. 2. - P. 841-853.

[7] Collingwood E. F., Lohwator A.J. The Theory of Cluster Sets. - Cambridge Tracts in Math. and Math. Physics 56. - Cambridge: Cambridge Univ. Press, 1966.

[8] Federer H. Geometric Measure Theory. - Berlin: Springer-Verlag, 1969.

[9] Gehring F.W. Rings and quasiconformal mappings in space // Trans. Amer. Math. Soc. - 1962. - 103, no. 3. - P. 353 - 393.

[10] Gehring F.W., Martio O. Quasiextremal distance domains and extension of quasiconformal mappings // J. Anal. Math. - 1985. - 45. - P. 181-206.

[11] Golberg A. Distortions of $p$-moduli of separating sets under mappings with finite integral dilatations // Bull. Soc. Sci. Lettres Lódź Sér. Rech. Déform. - 2003. - 40. - P. 41-51.

[12] Golberg A. Homeomorphisms with finite mean dilatations // Contemporary Math. - 2005. - 382. - P. 177-186.

[13] Golberg A. Coefficients of quasiconformality of ring domains // Proc. Inst. Math. NAS Ukraine. - 2004. - 1(3). - P. 77-86.

[14] Golberg A., Kud'yavin V.S. Mean coefficients of quasiconformality of pair of domains // Ukrain. Mat. Zh. - 1991. - 43(12). - P. 1709-1712 [in Russian]; translation in Ukrain. Math. J. - 1991. - 43. - P. 1594-1597.

[15] Goluzin G. M. Geometric Theory of Functions of a Complex Variable. - Transl. of Math. Monographs 26. - Providence: AMS, 1969.

[16] Gutlyanskit V., Ryazanov V., Srebro U., Yakubov E. The Beltrami Equations: A Geometric Approach. - Developments in Math. 26. - New York etc.: Springer, 2012.

[17] Heinonen J., Kilpelainen T., Martio O. Nonlinear Potential Theory of Degenerate Elliptic Equations. - Oxford-New York-Tokio: Clarendon Press, 1993.

[18] Hencl S., Koskela P. Lectures on mappings of finite distortion. - Lecture Notes in Math. 2096. - Cham: Springer, 2014.

[19] Ignat'ev A., Ryazanov V. Finite mean oscillation in the mapping theory // Ukr. Mat. Vis. - 2005. - 2, no. 3. - P. 395-417, 443 [in Russian]; transl. in Ukr. Math. Bull. - 2005. 2, no. 3. - P. 403-424, 443.

[20] Iwaniec T., Martin G. Geometric function theory and non-linear analysis. - Oxford: Oxford Univ. Press, 2001.

[21] Iwaniec T., Sbordone C. Riesz transforms and elliptic PDEs with VMO coefficients // J. Anal. Math. - 1998. - 74. - P. 183-212. 
[22] Iwaniec T., Sverak V. On mappings with integrable dilatation // Proc. Amer. Math. Soc. - 1993. - 118. - P. 181-188.

[23] John F., Nirenberg L. On functions of bounded mean oscillation. Comm. Pure Appl. Math. - 1961. - 14. - P. 415-426.

[24] Kovtonyuk D., Petkov I., Ryazanov V. On the boundary behaviour of solutions to the Beltrami equations // Complex Variables and Elliptic Equations. - 2013. - 58, no. 5. P. $647-663$.

[25] Kovtonyuk D.A., Petkov I.V., Ryazanov V.I., Salimov R.R. Boundary behavior and Dirichlet problem for Beltrami equations // Algebra and Analysis. - 2013. - 25, no. 4. P. 101-124 [in Russian]; transl in St. Petersburg Math. J. - 2014. - 25. - P. 587- 603.

[26] Kovtonyuk D.A., Ryazanov V.I. On boundaries of space domains // Proc. Inst. Appl. Math. \& Mech. NAS of Ukraine. - 2006. - 13. - P. 110 - 120.

[27] Kovtonyuk D.A., Ryazanov V.I. Toward the theory of lower Q-homeomorphisms // Ukr. Mat. Visn. - 2008. - 5, no. 2. - P. 159 - 184 [in Russian]; transl. in Ukrainian Math. Bull. - 2008. - 5, no. 2. - P. $157-181$.

[28] Kovtonyuk D., Ryazanov V. On the theory of mappings with finite area distortion // J. Anal. Math. - 2008. - 104. - P. 291-306.

[29] Kovtonyuk D., Ryazanov V. On the boundary behavior of generalized quasi-isometries // J. Anal. Math. - 2011. - 115. - P. 103-119.

[30] Kruglikov V.I. The existence and uniqueness of mappings that are quasiconformal in the mean. - In: Metric Questions of the Theory of Functions and Mappings, pp. 123-147. Kiev: Naukova Dumka, 1973 [in Russian].

[31] Kruglikov V.I. Capacities of condensors and quasiconformal in the mean mappings in space // Mat. Sb. - 1986. - 130(2). - P. 185-206 [in Russian].

[32] Kruglikov V.I., Miklyukov V.M. On classes of plane topological mappings with generalized derivatives. - In: Metric Questions of the Theory of Functions and Mappings, pp. 102-122. - Kiev: Naukova dumka, 1973 [in Russian].

[33] Kruglikov V.I., Paikov V.I. Capacities and prime ends of an $n$-dimensional domain // Dokl. Akad. Nauk Ukrain. SSR Ser. A. - 1987. - 5. - P. 10-13 [in Russian].

[34] KrushkaL' S.L. On mappings quasiconformal in the mean // Dokl. Akad. Nauk SSSR. 1964. - 157(3). - P. 517-519 [in Russian].

[35] KrushKaL' S.L. On the absolute integrability and differentiability of some classes of mappings of many-dimensional domains // Sib. Mat. Zh. - 1965. - 6(3). - P. 692-696 [in Russian].

[36] KrushkaL' S.L. On mappings $\varepsilon$-quasiconformal in the mean // Sib. Mat. Zh. - 1967. 8(4). - P. 798-806 [in Russian]. 
[37] KrushKal' S.L., KÜHnau R. Quasiconformal mappings, new methods and applications. Novosibirsk: Nauka, 1984 [in Russian].

[38] KUD'yavin V.S. A characteristic property of a class of $n$-dimensional homeomorphisms // Dokl. Akad. Nauk Ukrain. SSR ser. A. - 1990. - 3. - P. 7-9 [in Russian].

[39] KuD'YAVin V.S. Quasiconformal mappings and $\alpha$-moduli of families of curves // Dokl. Akad. Nauk Ukrain. - 1992. - 7. - P. 11-13 [in Russian].

[40] KuD'Yavin V.S. Estimation of the distortion of distances under mappings quasiconformal in the mean // Dinam. Sploshn. Sred. - 1981. - 52. - P. 168-171 [in Russian].

[41] Kud'yavin V.S. Local boundary properties of mappings quasiconformal in the mean. In: Collection of Scientific Works, Institute of Mathematics, pp. 168-171. - Novosibirsk: Siberian Branch of the Academy of Sciences of the USSR, 1981 [in Russian].

[42] KUD'YAVIN V.S. Behavior of a class of mappings quasiconformal in the mean at an isolated singular point // Dokl. Akad. Nauk SSSR. - 1984. - 277(5). - P. 1056-1058 [in Russian].

[43] KÜHnAu R. Über Extremalprobleme bei im Mittel quasiconformen Abbildungen // Lecture Notes in Math. - 1983. - 1013. - P. 113-124 [in German].

[44] Lelong-FerRAnd J. Representation conforme et transformations à integrale de Dirichlet bornée. - Paris: Gauthier-Villars, 1955.

[45] MaLy J. A simple proof of the Stepanov theorem on differentiability alsmost everywhere // Exposition Math. - 1999. - 17. - P. 59-61.

[46] Martio O., Ryazanov V., Srebro U., Yakubov E.: Q-homeomorphisms. Contemporary Math. - 2004. - 364. - P. 193-203.

[47] Martio O., Ryazanov V., Srebro U., Yakubov E.: On Q-homeomorphisms. Ann. Acad. Sci. Fenn. - 2005. - 30. - P. 49-69.

[48] Martio O., Ryazanov V., Srebro U., Yakubov E. Moduli in Modern Mapping Theory. - Springer Monographs in Mathematics. - New York etc.: Springer, 2009.

[49] Martio O., Rickman S., Vaisala J. Definitions for quasiregular mappings // Ann. Acad. Sci. Fenn. Ser. A1. Math. - 1969. - 448. - P. 1-40.

[50] Martio O., Ryazanov V., Vuorinen M. BMO and Injectivity of Space Quasiregular Mappings // Math. Nachr. - 1999. - 205. - P. 149-161.

[51] Martio O., Sarvas J. Injectivity theorems in plane and space // Ann. Acad. Sci. Fenn. Ser. A1 Math. - 1978/1979. - 4. - P. 384-401.

[52] Miklyukov V.M., Suvorov G.D. On existence and uniqueness of quasiconformal mappings with unbounded characteristics. - In: Investigations in the Theory of Functions of Complex Variables and its Applications. - Kiev: Inst. Math., 1972 [in Russian]. 
[53] NÄKki R. Boundary behavior of quasiconformal mappings in $n$-space // Ann. Acad. Sci. Fenn. Ser. A1. Math. - 1970. - 484. - P. 1-50.

[54] NÄKKI R. Prime ends and quasiconformal mappings // J. Anal. Math. - 1979. - 35. - P. $13-40$.

[55] Ohtsuka M. Extremal Length and Precise Functions. - Tokyo: Gakkotosho Co., 2003.

[56] Palagachev D.K. Quasilinear elliptic equations with VMO coefficients // Trans. Amer. Math. Soc. - 1995. - 347, no. 7. - P. 2481-2493.

[57] Perovich M. Isolated singularity of the mean quasiconformal mappings // Lect. Notes in Math. - 1979. - 743. - P. 212-214.

[58] Perovich M. Global homeomorphism of mappings quasiconformal in the mean // Dokl. Akad. Nauk SSSR. - 1976. - 230(4). - P. 781-784 [in Russian].

[59] Pesin I.N. Mappings quasiconformal in the mean // Dokl. Akad. Nauk SSSR. - 1969. 187(4). - P. 740-742 [in Russian].

[60] Rado T., Reichelderfer P.V. Continuous Transformations in Analysis. - Berlin etc.: Springer, 1955.

[61] Ragusa M.A. Elliptic boundary value problem in vanishing mean oscillation hypothesis // Comment. Math. Univ. Carolin. - 1999. - 40, no. 4. - P. 651-663.

[62] Reimann H.M., Rychener T. Funktionen Beschränkter Mittlerer Oscillation. - Lecture Notes in Math. 487. - Berlin etc.: Springer-Verlag, 1975.

[63] Reshetnyak Yu.G. Space mappings with bounded distortion. - Novosibirsk: Nauka, 1982; transl. in Translations of Mathematical Monographs, 73. - Providence, RI: Amer. Math. Soc., 1988.

[64] Rickman S. Quasiregular Mappings. - Berlin etc.: Springer-Verlag, 1993.

[65] Ryazanov V.I. On compactification of classes with integral restrictions on the Lavrent'ev characteristics // Sibirsk. Mat. Zh. - 1992. - 33(1). - P. 87-104 [in Russian]; transl. in Siberian Math. J. - 1992. - 33(1). - P. 70-86.

[66] RyaZAnov V.I. On quasiconformal mappings with measure restrictions // Ukrain. Mat. Zh. - 1993. - 45(7). - P. 1009-1019 [in Russian]; transl. in Ukrainю Math. J. - 1993. - 45(7). P. 1121-1133.

[67] Ryazanov V.I. On mappings that are quasiconformal in the mean // Sibirsk. Mat. Zh. 1996. - 37(2). - P. 378-388 [in Russian]; transl. in Siberian Math. J. - 1996. - 37(2). - P. 325-334. 
[68] Ryazanov V.I., Salimov R.R. Weakly flat spaces and bondaries in the mapping theory // Ukr. Mat. Vis. - 2007. - 4, № 2. - P. 199 - 234 [in Russian]; transl. in Ukrainian Math. Bull. - 2007. - 4, no. 2. - P. $199-233$.

[69] Ryazanov V., Salimov R., Srebro U., Yakubov E. On Boundary Value Problems for the Beltrami Equations // Contemporary Math. - 2013. - 591. - P. 211-242.

[70] Ryazanov V., Sevost'yanov E. Equicontinuons classes of ring $Q$-homeomorphisms // Sibirsk. Math. Zh. - 2007. - 48, № 6. - P. 1361-1376 [in Russian]; transl. in Siberian Math. J. - 2007. - 48, no. 6. - P. 1093-1105.

[71] Ryazanov V., Sevost'yanov E. Equicontinuity of mappings quasiconformal in the mean // Ann. Acad. Sci. Fenn. - 2011. - 36. - P. $231-244$.

[72] Ryazanov V., Srebro U., Yakubov E. On ring solutions of Beltrami equation // J. Anal. Math. - 2005. - 96. - P. 117-150.

[73] Ryazanov V., Srebro U., Yakubov E. To strong ring solutions of the Beltrami equations // Uzbek. Math. J. - 2009. - No. 1. - P. 127-137.

[74] Ryazanov V., Srebro U., Yakubov E. On strong solutions of the Beltrami equations // Complex Var. Elliptic Equ. - 2010. - 55, no. 1-3. - P. 219-236.

[75] Ryazanov V., Srebro U., Yakubov E. Integral conditions in the theory of the Beltrami equations // Complex Var. Elliptic Equ. - 2012. - 57, no. 12. - P. 1247-1270.

[76] Ryazanov V., Srebro U., Yakubov E. On integral conditions in the mapping theory // Ukrainian Math. Bull. - 2010. - 7, no. 1. - P. 73-87.

[77] SAKs S. Theory of the Integral. - New York: Dover Publications Inc., 1964.

[78] Sarason D. Functions of vanishing mean oscillation // Trans. Amer. Math. Soc. - 1975. 207. - P. 391-405.

[79] ShLYK V.A. On the equality between $p$-capacity and p-modulus // Sibirsk. Mat. Zh. - 1993. - 34, no. 6. - P. 216 - 221 [in Russian]; transl. in Siberian Math. J. - 1993. - 34, no. 6. - P. $1196-1200$.

[80] Stepanov W. Sur les conditions de l'existence de la differentielle totale // Mat. Sb. - 1925. - 32. - P. 511-526.

[81] Strugov Y.F. Compactness of the classes of mappings quasiconformal in the mean // Dokl. Akad. Nauk SSSR. - 1978. - 243(4). - P. 859-861 [in Russian].

[82] Strugov Y.F., Sychov A.V. On different classes of mappings quasiconformal in the mean // Vest. PANI. - 2002. - 7. - P. 14-19 [in Russian].

[83] Suvorov G.D. Generalized principle of the length and area in the mapping theory. - Kiev: Naukova Dumka, 1985 [in Russian]. 
[84] Suvorov G.D. The metric theory of prime ends and boundary properties of plane mappings with bounded Dirichlet integrals. - Kiev: Naukova Dumka, 1981 [in Russian].

[85] Uhlov A., Vodopyanov S. Mappings associated with weighted Sobolev spaces // Complex Anal. Dynam. Sys. III. Contemp. Math. - 2008. - 455. - P. 363-382.

[86] Ukhlov A., Vodopyanov S. Sobolev spaces and $(P, Q)$-quasiconformal mappings of Carnot groups // Siberian Math. J. - 1998. - 39. - P. 665-682.

[87] Vasil'ev A. Moduli of Families of Curves for Conformal and Quasiconformal Mappings. Lecture Notes in Math. 1788. - Berlin etc.: Springer-Verlag, 2002.

[88] VÄIsÄLÄ J. Lectures on n-Dimensional Quasiconformal Mappings. - Lecture Notes in Math. 229. - Berlin etc.: Springer-Verlag, 1971.

[89] Vodop'yanov S. Mappings with bounded distortion and with finite distortion on Carnot groups // Sibirsk. Mat. Zh. - 1999. - 40, no. 4. - P. 764-804; transl. in Siberian Math. J. 1999. - 40, no. 4. - P. 644-677.

[90] Vuorinen M. Conformal Geometry and Quasiregular Mappings. - Lecture Notes in Math. 1319. - Berlin etc.: Springer-Verlag, 1988.

[91] Whyburn G.Th. Analytic Topology. - Providence: AMS, 1942.

[92] Wilder R.L. Topology of Manifolds. - New York: AMS, 1949.

[93] Ziemer W.P. Extremal length and conformal capacity // Trans. Amer. Math. Soc. - 1967. - 126, no. 3. - P. $460-473$.

[94] Zorich V.A. Admissible order of growth of the characteristic of quasiconformality in the Lavrent'ev theory // Dokl. Akad. Nauk SSSR. - 1968. - 181. - P. [in Russian].

[95] Zorich V.A. Isolated singularities of mappings with unbounded distortion // Mat. Sb. 1970. - 81. - P. 634-638 [in Russian].

\section{Denis Kovtonyuk, Igor' Petkov and Vladimir Ryazanov,}

Institute of Applied Mathematics and Mechanics, National Academy of Sciences of Ukraine, 74 Roze Luxemburg Str., Donetsk, 83114, Ukraine, denis_kovtonyuk@bk.ru, igorpetkov@list.ru, vl.ryazanov1@gmail.com 